\title{
A Novel Resource Scheduling Algorithm to Improve TCP Performance for 3GPP LTE Systems
}

\author{
Peng Shang ${ }^{1}$, Yuhui Zeng ${ }^{2}$, Jinsong $\mathrm{Wu}^{1}$, Senior Member, IEEE, and Pei Xiao ${ }^{3}$, Senior Member, IEEE,
}

\begin{abstract}
The Long Term Evolution (LTE) may provide ubiquitous mobile broadband services with all IP architecture, however, the quality of service (QoS) of LTE systems is seriously affected by the network congestions, packet losses, jitters, latencies and other QoS issues in all IP networks. Thus it is valuable to investigate and design efficient resource scheduling algorithms to improve the performance of data services and enduser experiences. In this paper we propose an improved radio resource scheduling algorithm over the existing semi-continuous scheduling algorithm for the voice over Internet Protocol (VoIP) data packets. Through mapping the TCP (transmission control protocol) ACK (acknowledgement) packets into a higher priority logical channel, the probability of both the discarded ACK packets and congestions in the wireless channels are reduced. As the result, the scheme may avoid frequently opening the TCP congestion control mechanism. The simulation results have shown the advantages of our proposed algorithm, such as the RTT (Round-Trip Time) packet delay reduction, improved throughput, acceptable stability, desirable performance, and son on..
\end{abstract}

Index Terms-LTE, resource scheduling, QoS, TCP

\section{INTRODUCTION}

The Long Term Evolution (LTE), also known as Evolved Universal Terrestrial Radio Access (E-UTRA), has been the new generation of mobile broadband network technologies beyond GSM/UMTS/HSPA launched by the Third Generation Partnership Project (3GPP) standards organization [1]. Compared with $2 \mathrm{G}$ and $3 \mathrm{G}$ wireless technologies, LTE technologies are able to support IP(Internet Protocol)-based business and provides end to end quality of service $(\mathrm{QoS})$. Through the mechanisms of signaling control resource reservation and so on in the LTE radio layer, LTE could support the end to end QoS control in the business layer. However, network congestion, packet loss, jitter, latency and other QoS issues in the IP bearer network would seriously affect the QoS quality in the business layer [2]. Non-congestion IP network does not exist so that the key is how to maintain the business quality when congestions occur. The present TCP(transmission control protocol) congestion control mechanism is designed for the characteristics of wired network. Therefore, as the core of

\footnotetext{
${ }^{1}$ P. Shang and J. Wu are with Bell Laboratories, Shanghai 201206, China, email: shang158@gmail.com and wujs@ieee.org. ${ }^{2}$ Y. Zeng with Cisco Systems, Shanghai, China, email: zeng16@gmail.com. ${ }^{3} \mathrm{P}$. Xiao is with University of Surrey, UK, email: p.xiao@surrey.ac.uk.
}

the wireless resource management, it is a valuable task to investigate and design good resource scheduling algorithms in order to improve the TCP service performance and end-user experiences.

Radio resource management (RRM) is an indispensable component to achieve high data capacity and fast transfer rates [3]. The main task of RRM is to reasonably allocate the wireless resources to various packet services of wireless users in order to effectively improve the quality of service utilization and ensure the fairness for users. Radio resource management is a process of seeking the optimal solution between the variety of performance indicators and weights according to different requirements such as different QoS constraints of the multimedia industry [4]. It is difficult for a single resource allocation algorithm to consistently meet all performance requirements. Compared with the existing semicontinuous scheduling methodology of voice over Internet Protocol (VoIP) data packets in LTE system, we propose an improved scheme for wireless resource scheduling in LTE system, which is called mapping and conversion schemes for TCP acknowledgement packet. The proposed scheme maps the TCP packet to a higher-priority idle logical channel, so that it could reduce the occurrence of discarded ACK (acknowledgement) packets in the wireless channel and the probability of congestions and avoid the frequent use of TCP congestion control mechanism. Our simulation results show that the proposed scheme may reduce the RTT(Round-Trip Time) delay of the service and improve the efficiency of TCP acknowledgement. Moreover, it has some advantages in stability and performance.

The remainder of the paper is organized as follows. After a discussion of related issues and research of TCP protocol in Section II, Section III describes the mapping and conversion schemes for TCP acknowledgement packet in details. Simulation results are presented and discussed in Section IV. Finally, our conclusions are given in Section V.

\section{RELATED ISSUES AND RESEARCH OF TCP PROTOCOL}

\section{IN MOBILE NETWORK}

Although the simply implemented non-connected end to end packet switching could be used for Internet transmission, it can not guarantee reliable transmissions and deal with 
network congestions. With the control and feedback mechanisms, TCP protocol can achieve reliable transmission, flow and congestion control. According to the historical statistics, approximately $95 \%$ of the Internet traffic uses TCP/IP protocol. Without receiving acknowledgment packet within a certain time during the processes of the TCP protocol, the TCP sender would assume that a packet has lost as a result of network congestions, and would jump into the congestion control mode. TCP congestion control mechanism plays an important role in controlling network congestions. The TCP protocol was proposed to maintain the stability of the Internet and heterogeneity (router buffer spaces, bandwidths, delays, and so on), all the traffic based on the fair allocation of bandwidth, efficiency, and congestion control and other issues. Although TCP could provide a reliable and robust data communication for Internet, TCP protocol is designed for wired networks using a windowed protocol, which could face severe challenges in achieving the satisfactory error rate performance and avoid network congestion, and so on. With the widespread deployment of the mobile broadband networks, TCP performance becomes increasingly important in mobile broadband network. Mobile networks have higher latencies and higher error rates over wired networks, however, TCP senders would assume that the current packet losses are incurred by network congestions and jump into the congestion control mode to reduce the transmission rate.

The original design of the TCP protocol was based on the assumption that the physical links are stable. However, the wireless networks inherently could not guarantee the stability of the links due to the characteristics of fading channels. The ideal relevant solution is to design new transport layer protocols to adapt to the wireless fading channels. However, it is currently impossible to fully avoid the use of TCP due to the extensive adoptions and applications. Another way is to improve the performance based on the current TCP protocol with the aid of TCP-friendly mechanisms which could better adapt the wireless networks. In this paper, we investigate how to separately improve the throughput and the fairness [5] for wireless TCP transmissions.

Although it is well-known that the congestion control mechanisms could reduce the deterioration of network congestions, the congestion control mechanisms would increase the RTT latencies and reduce system throughput. In wireless networks, the main reason of TCP transmission timeout is the high loss ratio of data packets caused by the link errors rather than network congestions. Therefore, reducing the probability of discarded TCP packets and congestion in the wireless link is the key to improve the TCP performance in the mobile environments [6]. Under the considerations of implementation, costs, network compatibility and scalability, it is desirable to integrate the adaptive approaches with the existing standard
TCP protocol to allow the continuous uses of the large number of the existing mature TCP rate control mechanism [4].

\section{MAPPING AND CONVERSION SCHEMES FOR TCP ACKNOWLEDGEMENT PACKET}

\section{A. Design principle}

We first introduce the architecture and implementation of LTE QoS. LTE data link layer includes the following sublayers: Medium Access Control layer (MAC), radio link control (RLC) layer and Packet Data Convergence Protocol layer (PDCP) [7]. Each sub-layer interface has services access point (SAP) for communications. The SAP between the physical layer and the MAC(Media access control) layer is associated with the transmission channels, the SAP between MAC layer and RLC layer is associated with the logical channels, and the SAP between RLC layer and PDCP layer is associated with the radio bearer. The QoS in LTE systems follows the concept of the QoS for 3GPP SAE(System Architecture Evolution). The QoS targets, indicators and resources mapping and implementation process in the LTE/SAE system are much simpler than those in UMTS (Universal Mobile Telecommunications System) QoS [8]. The LTE SAE bearing for each different traffic is different. The traffic for the user is mapped to a different logical channel priority according to the different QoS requirement in PDCP sub-layer. Therefore, different QoS requirements determine the services and access order of PRB (Physical Resource Block) in the resource scheduling system.

In the TCP traffic, a separate ACK (acknowledgment packet) is used with a smaller size, and thus does not take up too much bandwidth for the voice packets in voice over Internet Protocol (VoIP). The specific resources are reserved for the VoIP traffic through the semi-continuous scheduling in LTE systems, and it has been put into a separate highest priority logical channel for transmission to ensure the voice quality. Therefore, we can map the separate ACK packet to the higher priority logical channel than the TCP data packet. As the result, when multiple users have a large business volume for FTP uploading and downloading at the same time, the TCP ACK packet has a higher priority to get resources and support and could not be easily blocked or wait for a longer delivery time, thereby the transmission delay RRT(Round-Trip Time) is reduced. Meanwhile, when the traffic is transmitted in a separate free channel, the channel conditions would be better, and the package would be also less prone to errors due to its smaller size, leading to the improved efficiency [9].

Based on the above ideas, we could see that the overall performance of TCP links could be improved if the cached data in the TCP sending window could be more timely identified and removed. Note that due to the lowest Type of Service (ToS) of TCP and under normal circumstances FTP 
(File Transfer Protocol) would be mapped to the lowest level of logical channels for transmissions. Therefore, regardless of which higher priority idle logical channel is mapped for the TCP ACK packet, it would be guaranteed to obtain the improved performance and QoS.

\section{B. Detailed algorithm}

In LTE systems, the eNB (UE) PDCP layer has a Mapping mechanism which would detect and maintain the ToS of the traffic for each newly established downlink (uplink) connection. The Mapping mechanism is used to determine the type of business and service level. Based on this information, the business packet system maps the packet to the corresponding logical channel, and also creates a static entry to store the ongoing task of the user between the business and the use of logical channels mapping, the priority information of distribution channel, and so on.

When the user has FTP businesses, the detection mechanism would determine whether there is a higher priority idle logical channel. If so, the individual ACK packets would be mapped to the logical channels for transmissions. If not, the system would select the normal channels for transmissions. When there are several idle logical channels, the highest priority channel is always being chosen. When the user has many businesses including FTP, HTTP (Hypertext Transfer Protocol), VoIP, and the channel for video conferences and emails is idle, the mapping mechanism would map the a single ACK packet to the video conference logical channel. At the receiver end, the separate ACK package received from each logical channel would be merged to the corresponding Radio Bearer by the ToS and then submitted to the top.

The brief charts of the mapping and conversion schemes for TCP acknowledgement packet are shown in Figs. 1 and 2. The sending process is described as follows.

\section{Step 1) When establishing a new data service, the algorithm would detect the current type of business and ToS, and determine whether the business is a new TCP packet. If yes, go to step 2; if not, go to Step 3; \\ Step 2) Map the packet to the corresponding logical chan- nel, record the current packet of information in the static entry, and return the information;}

Step 3) Check whether the packet is a separate TCP ACK packet, if not, move on to Step 2; if so, move to Step 4;

Step 4) Check the static entry and see whether there is a higher priority idle logical channel, if not, move on to Step 2; if there is, go to Step 5;

Step 5) Map the current business to the corresponding free logical channel, record the corresponding information in the static table, and return the corresponding information.

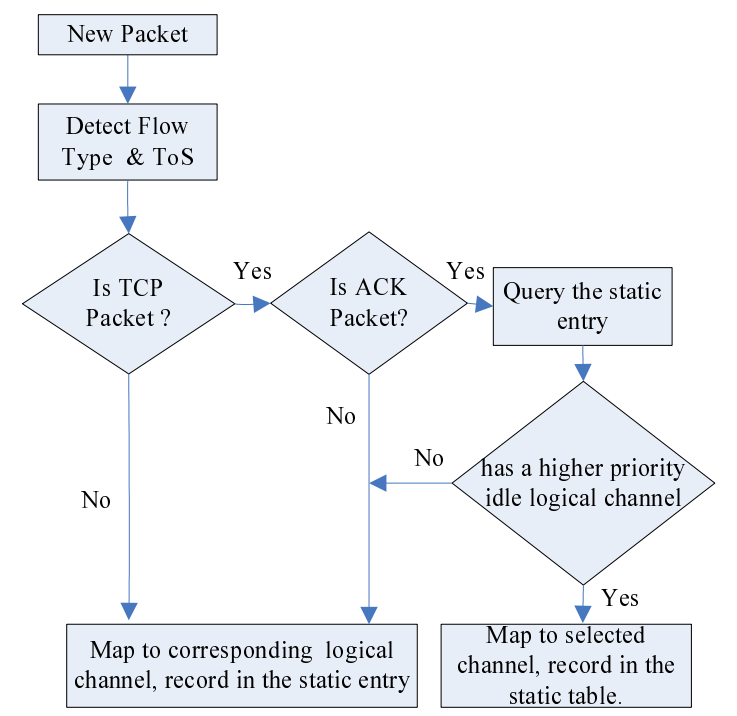

Fig. 1: Sending process for TCP ACK packet mapping

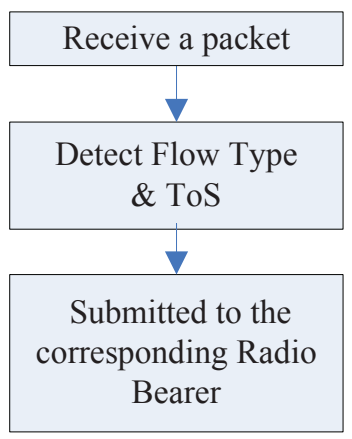

Fig. 2: Receiving process for TCP ACK packet mapping

Obviously, when the mapping mechanism detects the conflict in the logical channel for new establishment business and corresponding TCP ACK packets, the TCP ACK packet would be mapped to other lower-priority idle channel. Referring to the above example, if the user adds a new video service later, the ACK packet would be automatically mapped to the corresponding logical channel of Email service. In the receiver, the ACK packets from each logical channel would be detected, thus it would not affect the process of the TCP acknowledgement.

\section{Simulations AND Analysis}

In the simulation, we select two users, and each User has three types of business: VoIP, upstream FTP and downstream FTP. VoIP has the highest priority and the uplink and downlink FTP is mapped to different logical channels with the same priority. The size of uploading FTP file is $2 \mathrm{MB}$ (megabytes) and the size of downloading FTP file size is 3MB. We mainly compare the performance of our proposed scheme with that 
of the original scheme in terms of TCP throughput, RTT delay. In addition, we would like to examine whether the scheme has any impact on higher priority traffic, such as VoIP, video conferences. Individual uplink and downlink FTP ACK package is mapped to the channel below VoIP priority logical channels. Three operations are also established at the start of the simulation. The simulation results are presented as follows.

\section{A. Comparison of FTP throughput}

Figs. 3 and 4 show the throughput comparisons in FTP uplink and downlink for User 1 and 2. As shown in Fig. 3, it takes approximately 3.5 seconds to upload packets with the original scheme of User 1 , and it takes approximately 2.5 seconds with the proposed scheme. It takes approximately approximately 4 seconds to upload packets with original scheme of User 2 , and it takes approximately 3.5 seconds with the proposed scheme. It can be found that our modified scheme enables approximately $30 \%$ increase of the total average throughput compared with the original scheme.

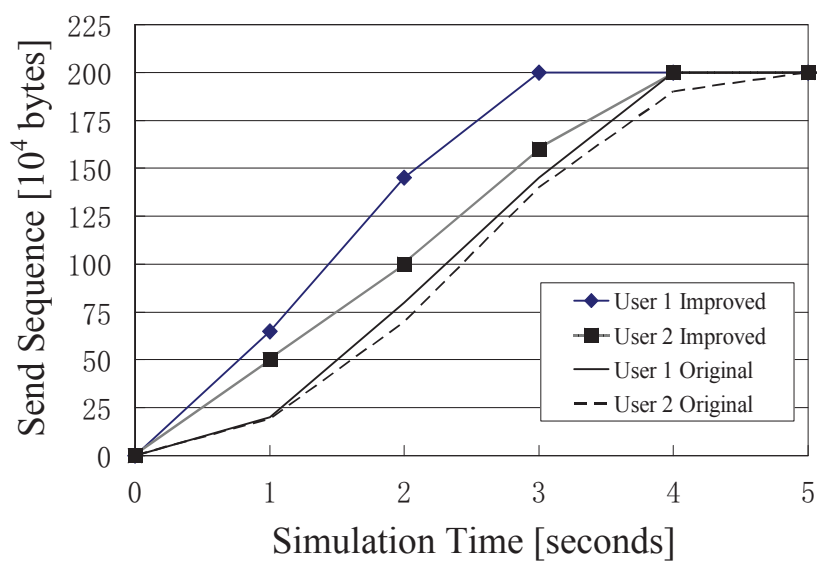

Fig. 3: Comparison Curve for FTP Uplink

Fig. 4 shows the throughput curve of FTP downlink. It takes approximately 6.5 seconds to download packets with the original scheme for User 1, and it takes approximately 5 seconds with our proposed scheme. It takes approximately 6 seconds to download packets with original scheme for User 2 , and it takes approximately 5 seconds with our proposed scheme. The average downloading throughput is increased by approximately $30 \%$ compared with the original scheme.

\section{B. Comparison of FTP RTT delay}

Figs. 5 and 5 show the curve of uplink and downlink RTT delays of User 1 and 2 with the original and the improved schemes.

It is shown in Fig. 5 that the time to achieve the stability state for the upstream FTP transmissions is approximately 3

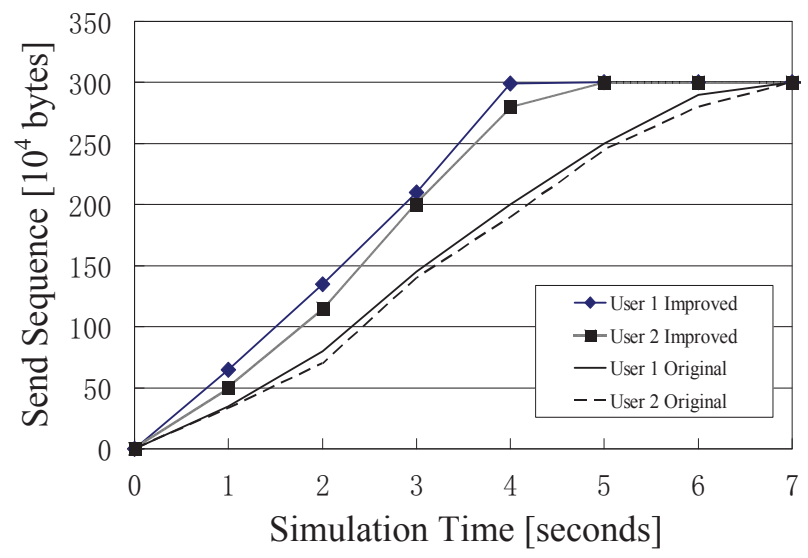

Fig. 4: Comparison Curve for FTP Downlink

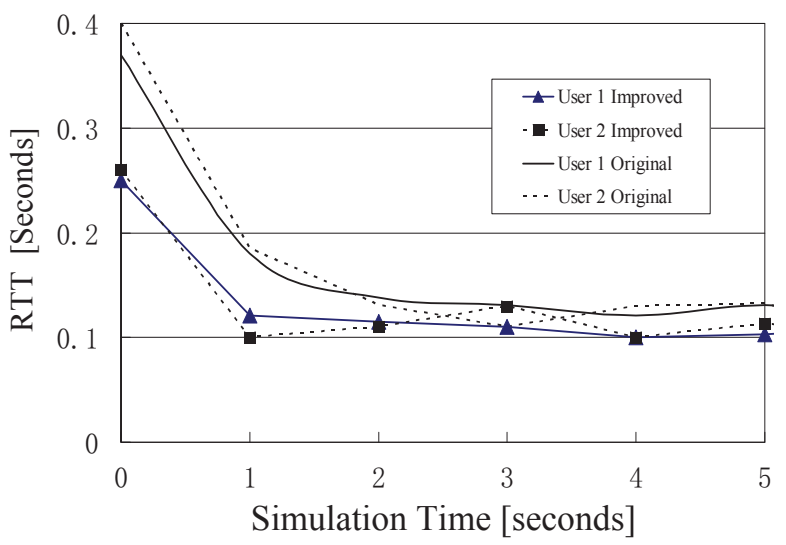

Fig. 5: Comparison curve for FTP Uplink Round-trip Delay

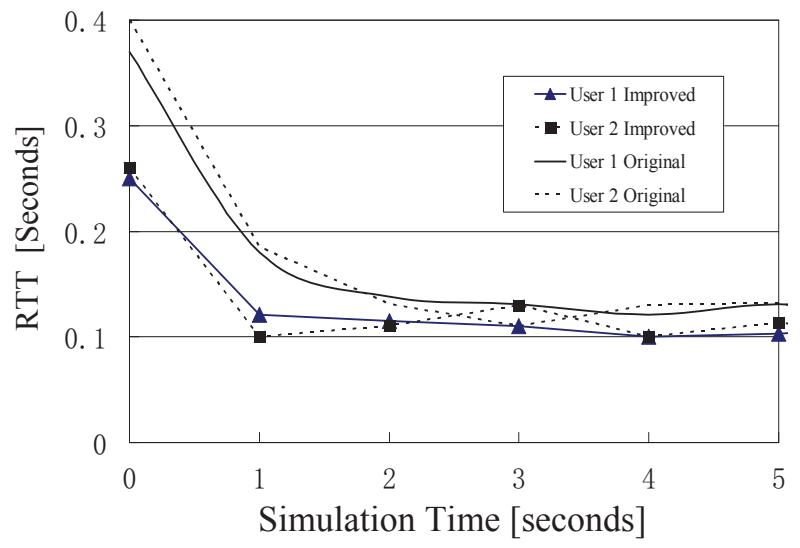

Fig. 6: Comparison curve for FTP Downlink Round-trip Delay 
to 5 seconds. During this period, the RTT delay of user 1 with original scheme is approximately 0.13 seconds and with improved scheme it is approximately 0.1 seconds. The RTT delay of user 2 with original scheme is approximately 0.14 seconds and with improved scheme is approximately 0.1 seconds. The time to achieve the stability state for the downstream FTP transmissions is approximately 5 to 6 seconds. During this period, the RTT delay of User 1 and User 2 with original scheme is approximately 0.14 seconds and with the improved scheme it is approximately 0.1 seconds. It could be concluded that, with the improved scheme, the RTT reduction is around $20 \sim 40$ milliseconds. The reason for the improvement the TCP ACK packet transmissions is to reduces the frequency using the TCP congestion control mechanisms.

\section{Point to point delay comparison of VoIP}

Figs. 7 and 8 show the curves of point to point VoIP delays of User 1 and 2 with original and improved schemes. It can be observed from these two figures that, for the VoIP end to end delays of User 1 and User 2, there basically is no differences between the original scheme and improved scheme, and most of the delays of ACK packets are around 10 to 30 milliseconds. It could be concluded that the improved scheme with the considerable stability would not affect the higher priority businesses.

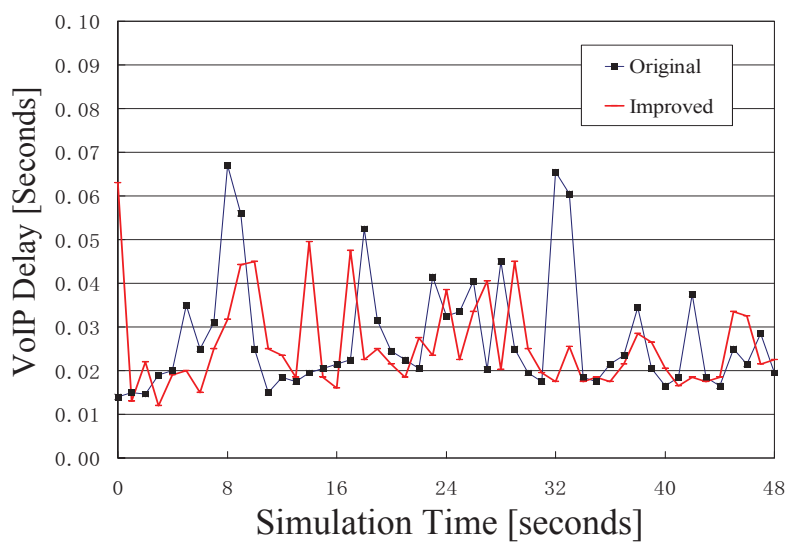

Fig. 7: VoIP Delay Distribution for User 1

\section{CONCLUSIONS}

In this paper, we analyze the related issues of existing TCP congestion control mechanisms applied to the mobile environments and point out that the characteristics in the mobile environments would reduce the TCP transmission efficiency. In order to adapt the QoS features of the LTE system, we have propose an algorithm called mapping and conversion schemes for TCP acknowledgement packets to improve the

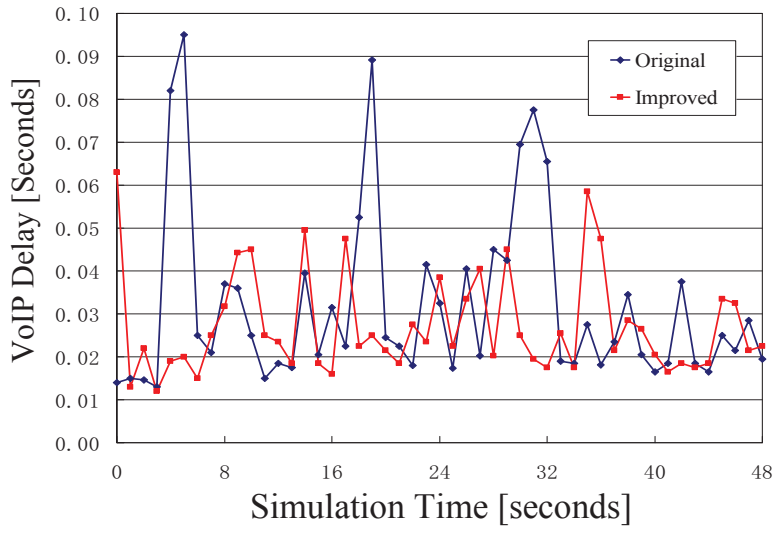

Fig. 8: VoIP Delay Distribution for User 2

system performance. The simulation results show that our proposed scheme could improve the overall performance of TCP operations without affecting the QoS performance for the transmissions of other users.

\section{REFERENCES}

[1] S. Sesia, I. Toufik, and M. Baker, "LTE - The UMTS Long Term Evolution: From Theory to Practice, 2nd ed.," Wiley, 2011.

[2] 3GPP TS 36.323 V9.0.0, "Packet Data Convergence Protocol (PDCP) Specification," pp. 7-10, Jan. 2010.

[3] 3GPP TS 36.401 V9.2.0, "Evolved Universal Terrestrial Radio Access Network (E-UTRAN); Architecture description,” pp. 18-21, Jan. 2010.

[4] M. Kaneko, P. Popovski, and J. Dahl, "Proportional Fairness in MultiCarrier System: Upper Bound and Approximation Algorithms," IEEE Communication Letters, vol. 10, No. 6, pp. 462-464, 2006.

[5] W. Richard Stevens, "TCP/IP Illustrated, Volume 1: The Protocols," Addison-Wesley, 1996.

[6] G. Barriac, J. Holtzman, "Introducing Delay Sensitivity into the Proportional Fair Algorithm for CDMA Downlink Scheduling," In Proc. IEEE International Symposium of Spread Spectrum Techniques and Applications, Prague, Czech Republic, pp. 652-656, 2002.

[7] 3GPP TS 36.300 V10.1.0, "Evolved Universal Terrestrial Radio Access (E-UTRA) and Evolved Universal Terrestrial Radio Access Network (EUTRAN); Overall description; Stage 2," pp. 23-26, Oct. 2010.

[8] K. N. Lau, "Analytical Framework for Multiuser Uplink MIMO SpaceTime Scheduling Design with Convex Utility Functions," IEEE Transactions on Wireless Communications, vol. 13, No. 5, pp. 1832-1843, Sept. 2004.

[9] Nilo Casimiro Ericsson, "Admission Control and Scheduling Based on Revenue-Maximum through Service Level Agreements," Sweden: Uppsala University, Oct. 2004. 